\title{
Desarrollo y evaluación de un prototipo de medición de color en vegetales frescos
}

\author{
Saúl Dussán-Sarria*, Alba M. Garzón-García, y Raúl E. Melo-Sevilla \\ Facultad de Ingeniería y Administración, Departamento de Ingeniería, Universidad Nacional de Colombia. Sede Palmira. \\ A.A. 237. Palmira, Valle del Cauca-Colombia. (e-mail: sdussan@unal.edu.co; almgarzonga@unal.edu.co; \\ remelos@unal.edu.co).
}

${ }^{*}$ Autor a quien debe ser dirigida la correspondencia

Recibido Feb. 21, 2019; Aceptado Abr. 15, 2019; Versión final Jun. 20, 2019, Publicado Feb. 2020

\section{Resumen}

El objetivo de este trabajo fue desarrollar y evaluar un prototipo de medición de color de vegetales frescos como alternativa económica frente a los equipos y técnicas comerciales. Se emplearon los sensores DHT11 para temperatura y TCS230-3200 para color, conectados a una tarjeta de adquisición de datos (Arduino Nano). La transferencia de información a un ordenador se estableció con los programas PXL-DAQ y Microsoft Exce ${ }^{\circledR}$ 2013, y las mediciones se registraron en el modelo de color RGB para su conversión al sistema CIE $\mathrm{L}^{*} \mathrm{a}^{*} \mathrm{~b}^{*}$. Se realizaron ensayos de comparación de medidas sobre la carta de color PANTONE con el prototipo, y colorímetro CR-400 Konica Minolta, determinándose el error medio entre las medidas. Se encontró un error medio promedio de $15,57 \%$ y $27,45 \%$ en las mediciones registradas por el prototipo de color y el colorímetro, respectivamente, lo cual evidenció que el uso del prototipo es una alternativa técnica apropiada de medición de color en vegetales frescos.

Palabras clave: equipo de medición; calidad de alimentos; sistema de adquisición de datos, colorímetro.

\section{Development and evaluation of a color measurement prototype in fresh vegetables}

\begin{abstract}
The aim of this work was to develop and evaluate a color measurement prototype in fresh vegetables as an economic alternative to equipment and commercial techniques. The DHT11 temperature and TCS230-3200 color sensors were used connected to a data acquisition card (Arduino Nano). The information transfer to a computer was established with the PXL-DAQ and Microsoft Excel ${ }^{\circledR} 2013$ software, and the measurements were recorded in RGB color model for its conversion to $\mathrm{CIE} \mathrm{L}^{*} \mathrm{a}^{*} \mathrm{~b}^{*}$ system. Comparison tests of measurements were carried out on PANTONE color chart with the prototype and a Konica Minolta CR-400 colorimeter, determining the average error between measurements. An average error of $15,57 \%$ and $27,45 \%$ was found in the measurements registered by the color prototype and the colorimeter, respectively, which showed that the use of prototype is an appropriate alternative technique for measuring color in fresh vegetables.
\end{abstract}




\section{INTRODUCCIÓN}

El color es un fenómeno físico y sensorial, captado por la percepción humana de la luz reflejada por un objeto y se ve afectado por el observador, el iluminante, la geometría óptica, el área, fondo, superficie, brillo y temperatura (Delmoro et al., 2010). Funciona como indicador de degradación y de la concentración de colorantes o pigmentos. Además, es el primer atributo que valora los consumidores y se considera fundamental en la elección de un producto, pues permite determinar la calidad de los alimentos y su idoneidad de forma rápida, precisa y objetiva (Mery y Pedreschi, 2005). La medición del color en los alimentos se puede efectuar con sistemas visuales humanos o instrumentos. En los sistemas visuales se hacen comparaciones con referencias de color bajo iluminación controlada o con colores estándar, implicando una inspección más especializada y entrenamiento de los observadores (Afshari-Jouybari y Farahnaky, 2011). Por otra parte, los equipos más utilizados son el colorímetro, espectrofotómetro y espectrocolorímetro (Garzón-García et al., 2018), e implican altos costos para su adquisición y calibración (Kirchner et al., 2018).

Ante esta situación, ultimadamente se ha empleado la visión computarizada (CVS) como tecnología para adquirir y analizar una imagen con computadores $u$ otras herramientas con el fin de obtener información o controlar procesos de forma económica y sencilla (Afshari-Jouybari y Farahnaky, 2011). Es un sistema que consta de cámaras digitales o de vídeo para la adquisición de imágenes mediante elementos sensibles a la luz, como un sensor CCD (dispositivo de carga acoplada) que integra a una matriz de filtros para que los objetos se vean a distintas intensidades (rojo $(R)$, verde $(G)$ y azul $(B)$ ) de acuerdo al balance de blancos. Las tres intensidades se combinan para producir la imagen digital y se convierten al sistema CIE L*a*b* (Mendoza et al., 2006).

Estudios como el de Afshari-Jouybari y Farahnaky (2011) evidencian el uso del software digital Photoshop para procesar las imágenes tomadas con una cámara digital, la cual estaba adaptada a un CVS. Los valores de $L^{*}, a^{*}$ y b* medidos con este método y un colorímetro Hunter tuvieron una alta correlación (coeficientes de determinación mayores a 0.98). En otra investigación, se evaluó el potencial de la regresión de máquinas de vectores de mínimos cuadrados (LS-SVM) para desarrollar un método basado en redes neuronales con el objetivo de medir el color de los alimentos en el sistema CIE $L^{*} a^{*} b^{*}$ mediante sistemas de visión computarizada. Tres modelos LS-SVM fueron validados, uno para cada variable de salida $\left(L^{*}, a^{*}\right.$ y $\left.b^{*}\right)$, utilizando los datos generados por una cámara digital (Romaniello et al., 2015). Rodríguez-Pulido et al. (2013) establecieron una metodología para mejorar la evaluación de colores heterogéneos en alimentos y su ilustración en el espació de color $\mathrm{CIE} \mathrm{L}^{*} \mathrm{a}^{*} \mathrm{~b}^{*}$, por medio de un algoritmo que clasifica las nubes de puntos en forma de elipsoides con un método de agrupación. A su vez, determinaron que el algoritmo puede ser útil para un estudiar la relación entre el color, la apariencia y la composición de los alimentos.

Hay que resaltar que la automatización de la medición del color ha permitido la integración de procesos dentro de las cadenas agroindustriales. Autores como Oblitas-Cruz y Castro-Silupu (2014) emplearon un sistema de visión computarizada con una nueva herramienta informática desarrollada en MATLAB 7.5, para evaluar el efecto del tiempo y la temperatura en el color del café tostado. El color fue medido en el sistema CIE L*a*b* y los datos se ajustaron a un diseño de superficie de respuesta. Con este estudio se logró la diferenciación de las tonalidades durante el tostado de café respecto a los parámetros de operación y optimizar el proceso. Ganganagowder y Kamath (2017) diseñaron un modelo supervisado de clasificación inteligente de productos alimenticios tales como galletas, cereales, verduras, nueces comestibles y otros, utilizando imágenes digitales. El algoritmo de selección de características basadas en correlación (CFS) y los pretratamientos de segunda derivada de las características morfológicas, de color y de textura se utilizaron para entrenar los modelos para la clasificación y la detección.

Notando la relevancia de disponer de instrumentos y equipos a nivel de planta piloto o laboratorio para llevar a cabo las mediciones de ciertos atributos en los vegetales frescos de forma automática, con incertidumbre tolerable y que no demanden grandes costos, el objetivo de este trabajo fue desarrollar un prototipo de medición de color económico, empleando sensores y tarjetas electrónicas, y validar su uso potencial mediante la comparación del error en las medidas con un colorímetro comercial, teniendo como referencia una carta de color especializada para alimentos.

\section{MATERIALES Y MÉTODOS}

Se detalla la construcción del prototipo para la medición de color y temperatura, la conversión de las mediciones de color del modelo RGB al sistema CIE L*a*b*, la forma en que se efectuaron los cálculos del error medio en la medida tanto para el prototipo como para el colorímetro, y la descripción del análisis estadístico. 


\section{Construcción del prototipo}

El prototipo de medición de color para uso en vegetales frescos fue construido y evaluado en el Laboratorio de Frutas y Hortalizas de la Universidad Nacional de Colombia, Sede Palmira. El prototipo consistió en una caja con dimensiones $20 \mathrm{~cm} \times 20 \mathrm{~cm} \times 20 \mathrm{~cm}$ y paredes de color negro, lo cual fue definido siguiendo las investigaciones efectuadas por Tarlak et al. (2016) y Lohumi et al. (2018). Se adaptaron el sensor de temperatura DHT11 en la base y el sensor de color TCS230-3200 que incluía LEDs, por encima del material a evaluar. Ambos sensores se conectaron a un Arduino Nano (Figura 1), el cual actuaba como tarjeta de adquisición de datos (DAQ). Mediante una conexión serial, se estableció la transferencia de los datos a un ordenador con la ayuda de un programa de fácil acceso llamado PXL-DAQ. Finalmente, se llevaron a un archivo en Microsoft Exce ${ }^{\circledR} 2013$ para realizar la conversión del sistema RGB al sistema CIE L*a*b* , como se muestra en la Figura 2. Fueron obtenidos 30 conjuntos de datos con cada referencia utilizada.

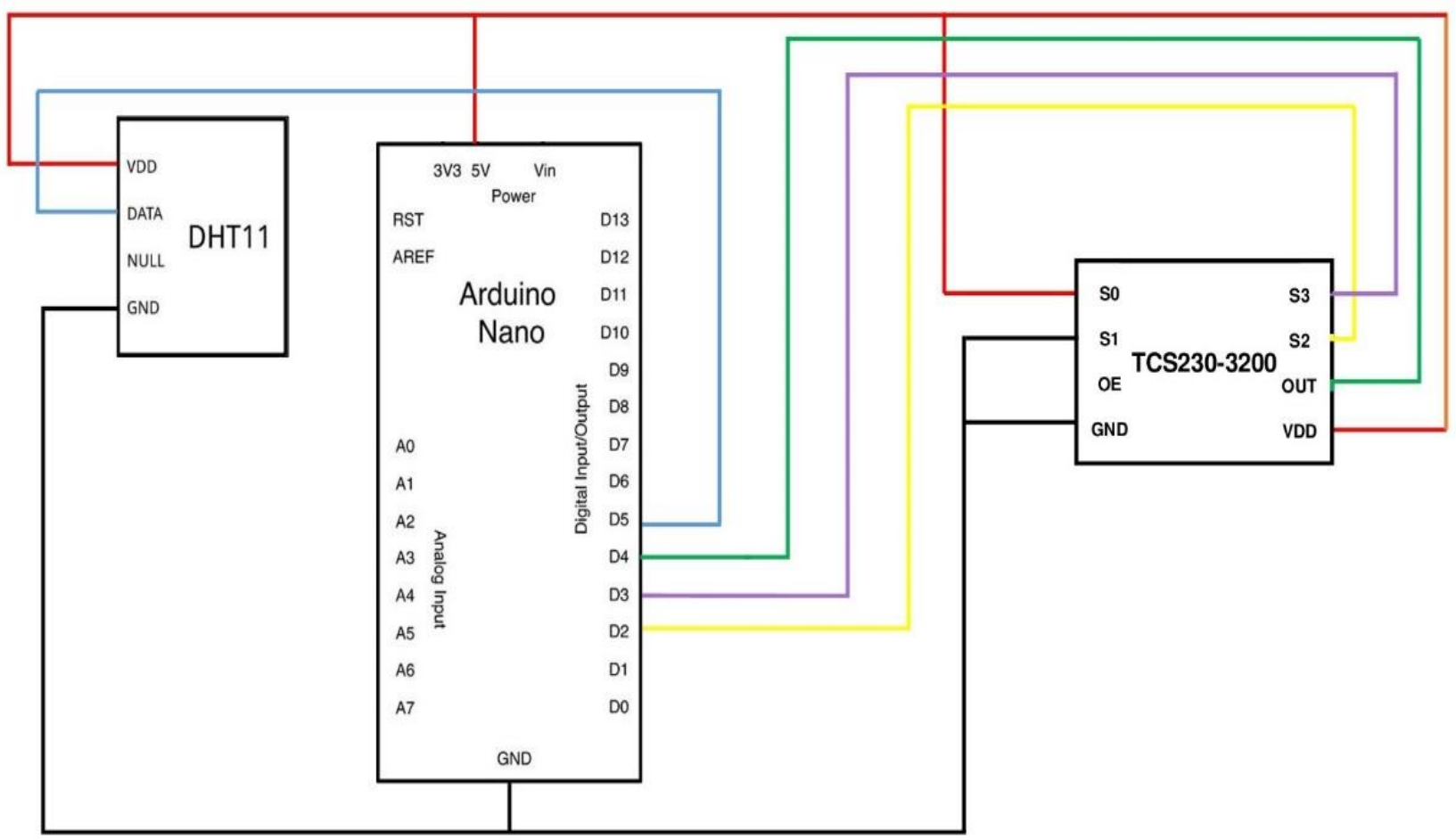

Fig. 1: Esquema de conexión entre la tarjeta de adquisición de datos (Arduino Nano) y el sensor de temperatura DHT11 en la base y el sensor de color TCS230-3200

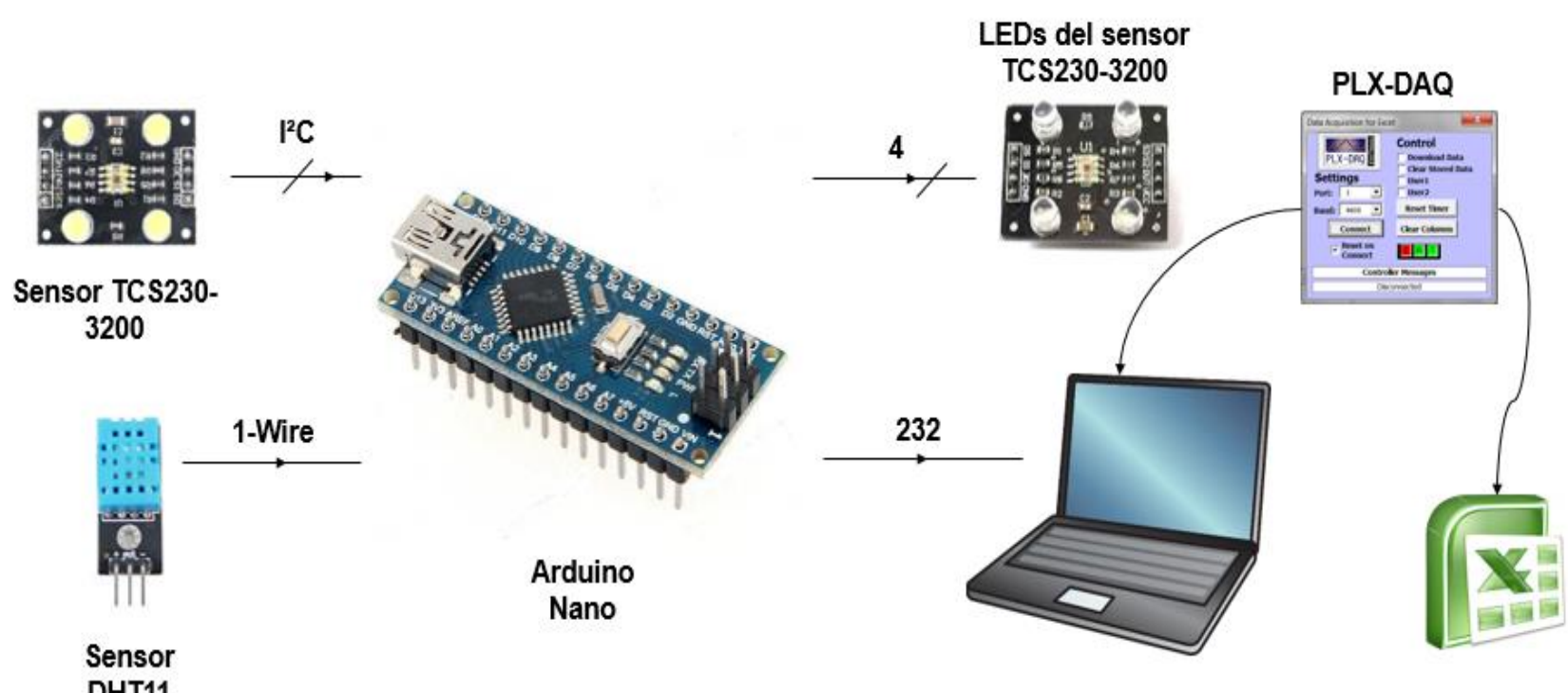

DHT11

Fig. 2: Diagrama de bloques para la medición de color con el prototipo desarrollado 


\section{Conversión del sistema RGB a CIE $L^{*} a^{*} b^{*}$}

El sensor TCS230-3200 detectó las medidas de color en el modelo de color RGB y se convirtieron al sistema CIE $L^{*} a^{*} b$, el cual es comúnmente usado para la medición de color en los alimentos. El proceso inició corrigiendo los valores $R G B(R=r o j o, G=v e r d e, B=a z u l)$ y transformándolos en valores $R G B$, para definirlos a partir de funciones de mapeo de codificación no uniforme (Mendoza et al., 2006). Luego, dichos valores fueron convertidos al sistema CIE XYZ y se usaron cálculos con coeficientes para un observador $2^{\circ}$ e iluminante D65 $\left(X_{n}=95.03 ; Y_{n}=100 ; Z_{n}=108.88\right)$. Finalmente, se obtuvieron las mediciones en el sistema CIE L*a*b* (Saldaña et al., 2014; Garzón-García et al., 2018).

Con las Ecuaciones 1, 2 y 3, obteniendo la primera variación.

$$
\begin{aligned}
\operatorname{VarR} & =\mathrm{R} / 255 \\
\operatorname{VarG} & =\mathrm{G} / 255 \\
\operatorname{VarB} & =\mathrm{B} / 255
\end{aligned}
$$

Se consideraron las condiciones a) y b) y se aplicaron las ecuaciones 4 a la 9 .

a) Si VarR; VarG; VarB $\leq 0.404045$ entonces,

$$
\begin{aligned}
& \mathrm{r}=\operatorname{VarR} / 12.92 \\
& \mathrm{~g}=\operatorname{VarG} / 12.92 \\
& \mathrm{~b}=\operatorname{VarB} / 12.92
\end{aligned}
$$

b) Si VarR; VarG; VarB >0.404045 entonces,

$$
\begin{aligned}
& \mathrm{r}=\left(\frac{\operatorname{VarR}+0.055}{1.055}\right)^{2.4} \\
& \mathrm{~g}=\left(\frac{\operatorname{VarG}+0.055}{1.055}\right)^{2.4} \\
& \mathrm{~b}=\left(\frac{\operatorname{VarB}+0.055}{1.055}\right)^{2.4}
\end{aligned}
$$

Se efectuó la conversión de los parámetros RGB al sistema CIE XYZ.

$$
\left[\begin{array}{l}
X \\
Y \\
Z
\end{array}\right]=100 \times\left[\begin{array}{lll}
0.4124 & 0.3576 & 0.1805 \\
0.2126 & 0.7152 & 0.0722 \\
0.0193 & 0.1192 & 0.9505
\end{array}\right]\left[\begin{array}{l}
\mathrm{r} \\
\mathrm{g} \\
\mathrm{b}
\end{array}\right]
$$

Teniendo las condiciones a) y b) y se aplicaron las ecuaciones 11 a la 16 para la conversión del sistema CIE $X Y Z$ al CIE L*a*b*.

a) Si $\frac{X}{X_{n}} ; \frac{Y}{Y_{n}} ; \frac{Z}{Z_{n}}>0.008856$ entonces;

$$
\begin{aligned}
& \operatorname{VarX}=\left(\frac{X}{X_{n}}\right)^{1 / 3} \\
& \operatorname{VarY}=\left(\frac{Y}{Y_{n}}\right)^{1 / 3} \\
& \operatorname{VarZ}=\left(\frac{Z}{Z_{n}}\right)^{1 / 3}
\end{aligned}
$$

b) $\quad$ Si $\frac{X}{X_{n}} ; \frac{Y}{Y_{n}} ; \frac{Z}{Z_{n}} \leq 0.008856$ entonces;

$$
\begin{aligned}
& \operatorname{VarX}=\left(7.787 \times \frac{\mathrm{X}}{\mathrm{X}_{\mathrm{n}}}\right)+\frac{16}{116} \\
& \operatorname{VarY}=\left(7.787 \times \frac{\mathrm{Y}}{\mathrm{Y}_{\mathrm{n}}}\right)+\frac{16}{116}
\end{aligned}
$$




$$
\operatorname{VarZ}=\left(7.787 \times \frac{\mathrm{Z}}{\mathrm{Z}_{\mathrm{n}}}\right)+\frac{16}{116}
$$

Los valores del sistema CIE L*a*b* se encontraron con las ecuaciones 17,18 y 19.

$$
\begin{aligned}
& \mathrm{L} *=(116 \times \operatorname{VarY})-16 \\
& \mathrm{a} *=500 \times(\operatorname{VarX}-\operatorname{VarY}) \\
& \mathrm{b} *=200 \times(\operatorname{Var} Y-\operatorname{Var} Z)
\end{aligned}
$$

\section{Determinación del error medio en la medida}

Siguiendo lo planteado por Mendoza et al. (2006), fueron considerados como referencia los valores $L^{*}$, $a^{*}$ y $b^{*}$ de la carta de color PANTONE los cuales fueron comparados, a través del error medio, con los valores $L^{*}$, $a^{*}$ y $b^{*}$ obtenidos por el prototipo y un colorímetro comercial. La carta de color ha sido utilizada en diversos trabajos para identificar el color en vegetales frescos siendo un estándar en la comparación de diferentes métodos de medición (Delmoro et al., 2010; Padrón et al, 2012; Newslow, 2014). Esta carta de color posee la descripción del color en el modelo CYMK, de carácter sustractivo y con las siglas de Cyan-Magenta-Yellowblack, (cian-magenta-amarillo-negro), el blanco resulta de la ausencia de todos los colores, mientras que el negro se obtiene sumándolos todos (Vallina, 2010). Los colores evaluados en la carta de color fueron Yellow C (C: 0, M: 0, Y: 100, K: 0), Orange 021 C (C: 0, M: 51, Y: 87, K: 0) y un blanco navajo, 1205 C (C: 0, M: 0, Y: $27, \mathrm{~K}: 0)$ que corresponden a los valores de las coordenadas $L^{*}, a^{*} y b^{*}$ resultantes de la conversión en línea con EasyRGB (Tabla 1).

Tabla 1: Valores de las coordenadas de color de referencia en el sistema CIE L*a*b*

\begin{tabular}{|c|c|c|c|}
\hline Color & $\mathrm{L}^{*}$ & $\mathrm{a}^{*}$ & $\mathrm{~b}^{*}$ \\
\hline Yellow C & 97 & -22 & 94 \\
\hline Orange 021 C & 67 & 45 & 67 \\
\hline 1205 C & 99 & -10 & 33 \\
\hline
\end{tabular}

En la carta de color fueron medidos los colores previamente mencionadas utilizando el prototipo construido y el colorímetro CR-400 Konica Minolta. El colorímetro cuenta con un software denominado Espectro Magic NX el cual registra de forma directa los valores de las coordenadas $L^{*}, a^{*} y b^{*}$. Fue calibrado con un plato o placa reflectora blanca $\left(L^{*}=97.58, a^{*}=0.16\right.$ y $\left.b^{*}=1.71\right)$, a un ángulo de observación de $2^{\circ}$ e iluminante $D 65$. Los parámetros determinados fueron $L^{*}\left(L^{*}=0\right.$ [negro] y $L^{*}=100$ [blanco]), $a^{*}\left(-a^{*}=\right.$ verde y $+a^{*}=$ rojo $)$ y $b^{*}\left(-b^{*}=a z u l\right.$ $y+b^{*}=$ amarillo).

\section{Análisis estadístico}

Se realizó un análisis ANOVA de un factor y Tukey en Microsoft Exce ${ }^{\circledR} 2013(\alpha=0,05)$, para evaluar si existían diferencias significativas entre las mediciones en el colorímetro y el prototipo evaluado. Inicialmente fue calculado el error porcentual entre los valores de las coordenadas $\mathrm{L}^{*}, \mathrm{a}^{*} \mathrm{y} \mathrm{b}^{*}$ con la ecuación 20 , donde $e_{n}$ el error porcentual de una coordenada del sistema CIE $L^{*} a^{*} b^{*}, n_{c}$ la coordenada del sistema CIE $L^{*} a^{*} b^{*}$ obtenida con la carta de color PANTONE y $\mathrm{n}_{\mathrm{p}}$ la coordenada del sistema CIE $\mathrm{L}^{*} \mathrm{a}^{*} \mathrm{~b}^{*}$ obtenida por la medición con el colorímetro o con el prototipo.

$$
\mathrm{e}_{\mathrm{n}}=\left[\frac{\mathrm{n}_{\mathrm{c}}-\mathrm{n}_{\mathrm{p}}}{\mathrm{n}_{\mathrm{c}}}\right] \times 100
$$

Consecuentemente, con la ecuación 21 se halló el error medio para los valores obtenidos con el colorímetro y el prototipo al conocer el error porcentual en las tres coordenadas $\mathrm{CIE} \mathrm{L}^{*} \mathrm{a}^{*} \mathrm{~b}^{*}$ en cada repetición (AfshariJouybari y Farahnaky, 2011). Siendo è el error medio expresado como porcentaje, $\mathrm{e}_{\mathrm{L}^{*}} \mathrm{el}$ valor del error porcentual en la coordenada $L^{*}, e_{b *}$ el valor del error porcentual en la coordenada $b^{*}$ y $e_{a *}$ el valor del error porcentual en la coordenada $a^{*}$, este procedimiento se realizó a cada uno de los 30 grupos de datos obtenidos.

$$
\overline{\mathrm{e}}=\frac{\mathrm{e}_{\mathrm{L} *}+\mathrm{e}_{\mathrm{a} *}+\mathrm{e}_{\mathrm{b} *}}{3}
$$

\section{RESULTADOS Y DISCUSIÓN}

Se exponen los resultados y discusión en las subsecciones mencionadas a continuación: mediciones con el prototipo y el colorímetro, y determinación del error medio en la medida. 


\section{Mediciones con el prototipo y el colorímetro}

Según Kirchner et al. (2018), los equipos convencionales para la medición del color en los alimentos generalmente tienen precios muy elevados, superando el presupuesto de las pequeñas empresas y consumidores. En consecuencia, se han dispuesto varios "instrumentos de color" mediante el procesamiento de imágenes digitales de cámaras, empleando el modelo RGB para medir el color de un objeto. Algunos de los inconvenientes del uso de la cámara digital es la dependencia de la luz ambiental para la medición y la segmentación de las imágenes en softwares (Photoshop e ImageJ) o funciones de otros programas como MATLAB.

En comparación a lo expresado anteriormente, la medición de color con el prototipo es dinámica, porque se evita el uso de un sistema de iluminación, ya que el sensor de color tiene dispositivos LED integrados. Tampoco se ve necesario segmentar imágenes o usar programas con licencia, porque dicho sensor capta las mediciones directamente en el modelo de color RGB y el sistema de adquisición de datos se planteó con un código fácil de programar y softwares libres. Una de las ventajas más significativas que tiene el diseño se basa en un principio de los instrumentos de medición de color en los alimentos propuesto por Brimelow y Joshi (2001), y es la mínima preparación de la muestra, tal como se muestra en la descripción de la cinética de la pérdida de la luminosidad de las rodajas de la raíz reservante de yacón empleando el prototipo, al exponer este material vegetal a temperatura ambiente después de su refrigeración (Garzón-García et al., 2018).

Por otro lado, se nota que existe una diferencia significativa entre los valores de $L^{*}$, $a^{*}$ y $b^{*}$ que fueron obtenidos por medio de la carta de color, el colorímetro y el prototipo para los colores Yellow C, Orange 021 C y $1205 \mathrm{C}(\alpha=0.05)$. Lo anteriormente expuesto se ilustra en la Tabla 2 , en la cual de acuerdo a los resultados de la prueba Tukey, las medias que no comparten una letra son significativamente diferentes $(p<0.05)$, a excepción de la coordenada $b^{*}$ en el color 1205 para la carta de color y el colorímetro. Afshari-Jouybari y Farahnaky (2011) utilizaron el software Photoshop con la idea de proporcionar una forma más versátil para evaluar el color en los alimentos, integrando una cámara digital para adquirir imágenes y a pesar de haber encontrado una alta correlación entre las medidas Hunter $L^{*} a^{*} b^{*}$ y el Photoshop, los valores de las coordenadas de color también presentaron diferencias significativas.

Tabla 2: Valores de las coordenadas del sistema CIE L*a*b encontradas para los colores de la tabla de color PANTONE, el colorímetro y el prototipo de medición de color

\begin{tabular}{|c|c|c|c|c|}
\hline \multirow[b]{2}{*}{ Color } & \multirow[b]{2}{*}{ Coordenada } & \multicolumn{3}{|c|}{ Instrumento } \\
\hline & & $\begin{array}{l}\text { Carta de color } \\
\text { PANTONE }\end{array}$ & Colorímetro & $\begin{array}{l}\text { Prototipo de medición } \\
\text { de color }\end{array}$ \\
\hline \multirow{3}{*}{ Yellow C } & $L^{*}$ & $97^{a}$ & $83,14^{c} \pm 0,36$ & $89,21^{\mathrm{b}} \pm 0,84$ \\
\hline & $a^{*}$ & $-22^{c}$ & $-9,06^{\mathrm{a}} \pm 0,04$ & $-15,29^{b} \pm 0,88$ \\
\hline & $b^{*}$ & $94^{a}$ & $67,10^{\circ} \pm 0,33$ & $86,78^{b} \pm 0,66$ \\
\hline \multirow{3}{*}{ Orange $021 \mathrm{C}$} & $L^{*}$ & $67^{a}$ & $58,60^{c} \pm 0,03$ & $65,25^{\mathrm{b}} \pm 1,13$ \\
\hline & $a^{*}$ & $45^{\mathrm{b}}$ & $47,40^{a} \pm 0,06$ & $40,65^{\circ} \pm 1,58$ \\
\hline & $b^{*}$ & $67^{a}$ & $41,12^{\mathrm{c}} \pm 0,05$ & $63,60^{b} \pm 2,24$ \\
\hline \multirow{3}{*}{$1205 \mathrm{C}$} & $L^{*}$ & $99^{\mathrm{a}}$ & $83,06^{b} \pm 0,42$ & $92,52^{b} \pm 0,95$ \\
\hline & $a^{*}$ & $-10^{b}$ & $-2,87^{a} \pm 0,02$ & $-15,67^{\mathrm{c}} \pm 0,87$ \\
\hline & $b^{*}$ & $33^{b}$ & $33,50^{b} \pm 0,27$ & $37,67^{a} \pm 1,13$ \\
\hline
\end{tabular}

De manera representativa, las tonalidades captadas a partir de los promedios de las medidas de los instrumentos son ilustradas en la Figura 3, por medio de EasyRGB. Se notó a simple vista mayor similitud entre las medidas del prototipo y la carta de color para los colores Yellow C y Orange $021 \mathrm{C}$. Los colores captados por el colorímetro se observan con menor luminosidad $\left(L^{*}\right)$ y el incremento de la coordenada $a^{*}$ (tonos rojizos). Estos tonos rojizos se explican porque los colores evaluados se encuentran en un espectro visible en el que se emiten tonalidades rojas y, además, hubo una pérdida de saturación debido a la disminución del parámetro L*. Lo anterior es congruente con los datos y comparaciones reportados en la Tabla 2. 


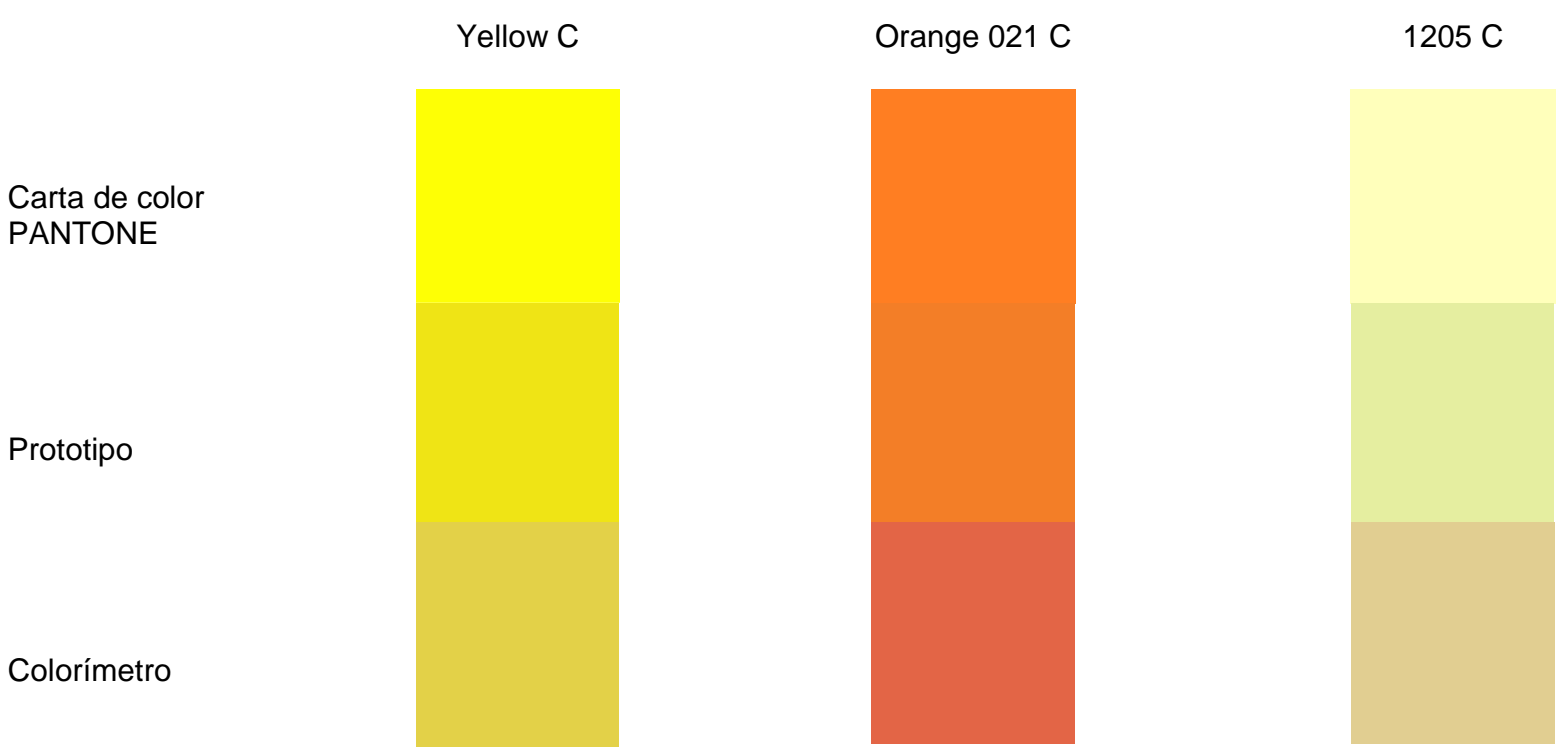

Fig. 3: Ilustración de comparación de los métodos de medición de color

\section{Determinación del error medio en la medida}

En la Tabla 3 se observan los resultados de los valores de los errores porcentuales y errores medios entre el colorímetro y la carta PANTONE, así como entre el prototipo y la carta PANTONE. Con excepción de los errores porcentuales de la coordenada $b^{*}$ en Orange $021 \mathrm{C}$ y la coordenada $\mathrm{a}^{*}$ en el $1205 \mathrm{C}$, los errores medios entre el prototipo y la carta de color fueron significativamente menores que los encontrados entre el colorímetro y la carta de color. Para los colores medidos Yellow C, Orange $021 \mathrm{C}$ y $1205 \mathrm{C}$, los errores medios entre el prototipo y la carta de color fueron $15,40 \%, 6,11 \%$ y $25,20 \%$ respectivamente correspondiendo a un valor de error medio de $15,57 \%$, mientras que entre el colorímetro y la carta de color fueron $33,90 \%$, $18,83 \%$ y $29,62 \%$ respectivamente correspondiendo a un valor de error medio de $27,45 \%$. Este error medio registrado por el colorímetro posiblemente se debió a que el colorímetro utilizado es un instrumento manual portátil y las mediciones se realizaron en un ambiente abierto no controlado, con distintas fuentes de iluminación, objetos alrededor y, la posición y tamaño del instrumento pudieron generar sombra en la carta de color en las lecturas. Por otro lado, el prototipo evaluado permite eliminar interferencias del medio en las mediciones y la posición del sensor de color puede ajustarse para lograr una mayor precisión en las lecturas.

Tabla 3: Valores de los errores medios (\%) entre los valores de las coordenadas CIE L*a*b de los colores Yellow C, Orange 021 C y 1205 C obtenidos entre el colorímetro y el prototipo con referencia a la carta PANTONE

\begin{tabular}{|c|c|c|c|}
\hline \multirow{3}{*}{ Color } & Tipo de error (\%) & \multicolumn{2}{|c|}{ Instrumento } \\
\cline { 2 - 4 } & $\mathrm{e}_{\mathrm{L} *}$ & $14,29 \pm 0,37$ & Prototipo de medición de color \\
\cline { 2 - 4 } & $\mathrm{e}_{\mathrm{b} *}$ & $58,81 \pm 0,19$ & $8,03 \pm 0,87$ \\
\cline { 2 - 4 } & $\mathrm{e}_{\mathrm{a} *}$ & $28,61 \pm 0,35$ & $30,49 \pm 3,98$ \\
\cline { 2 - 4 } & $\overline{\mathrm{e}}$ & $33,90 \pm 0,28$ & $7,69 \pm 0,70$ \\
\hline \multirow{3}{*}{ Yellow C } & $\mathrm{e}_{\mathrm{L} *}$ & $12,53 \pm 0,05$ & $15,40 \pm 1,83$ \\
\cline { 2 - 4 } & $\mathrm{e}_{\mathrm{b} *}$ & $5,32 \pm 0,14$ & $2,71 \pm 1,49$ \\
\cline { 2 - 4 } & $\mathrm{e}_{\mathrm{a} *}$ & $38,62 \pm 0,07$ & $9,67 \pm 3,52$ \\
\cline { 2 - 4 } & $\overline{\mathrm{e}}$ & $18,83 \pm 0,08$ & $5,95 \pm 1,00$ \\
\hline \multirow{3}{*}{ Orange 021 C } & $\mathrm{e}_{\mathrm{L} *}$ & $16,10 \pm 0,42$ & $6,11 \pm 0,77$ \\
\hline & $\mathrm{e}_{\mathrm{b} *}$ & $71,25 \pm 0,19$ & $4,75 \pm 1,09$ \\
\cline { 2 - 4 } & $\mathrm{e}_{\mathrm{a} *}$ & $1,51 \pm 0,82$ & $56,71 \pm 8,70$ \\
\cline { 2 - 4 } & $\overline{\mathrm{e}}$ & $29,62 \pm 0,10$ & $14,15 \pm 3,43$ \\
\cline { 2 - 4 } & & & $25,20 \pm 2,80$ \\
\hline
\end{tabular}

Saldaña et al. (2013) realizaron mediciones de los parámetros de color $L^{*}, a^{*}$ y $b^{*}$ a 30 cartulinas de colores con un sistema de visión computarizada (CVS), el cual consistía en una videocámara y una interfaz gráfica de usuario (GUIDE) de MATLAB. Estas mediciones se compararon con las determinaciones efectuadas para el mismo material con un colorímetro, y se observaron errores cercanos al $5 \%$. Con esto, se estableció que 
el algoritmo utilizado por el CVS para la conversión de unidades del modelo de color RGB a CIE L*a*b* es fiable. Este mismo algoritmo fue empleado para realizar las conversiones de las medidas del prototipo, logrando un diseño económico, de fácil uso y un error medio menor al encontrado usando el equipo comercial.

\section{CONCLUSIONES}

De acuerdo a los resultados y sus análisis, se obtienen las siguientes conclusiones, sobre el prototipo de medición de color en vegetales frescos: 1) el prototipo desarrollado mediante la integración de componentes como sensores y tarjeta de adquisición de datos y softwares libres, permite evaluar el color de forma distinta; 2) el error medio registrado por el prototipo fue de $15,57 \%$, valor inferior al registrado por el colorímetro comercial que fue de $27,45 \%$; 3 ) el prototipo diseñado y construido para la medición de color presenta un costo considerablemente menor si comparado con los costos de los equipos comerciales; 4) se hace necesario realizar evaluaciones comparativas con otros sistemas e instrumentos utilizados para la medición de color.

\section{REFERENCIAS}

Afshari-Jouybari, H. y A. Farahnaky, Evaluation of Photoshop Software Potential for Food Colorimetry, doi: 10.1016/j.jfoodeng.2011.02.034, J. Food Eng.,106(2), 170-175 (2011).

Brimelow, C. J. B. y P. Joshi, Color Measurement of Foods by Color Reflectance; en Instrumentation and Sensors for the Food Industry por E. Kress-Rogers y J. B. Brimelow, $2^{\mathrm{a}}$ Ed., pp 8-116, Woodhead Publishing Limited and CRC Press LLC, Boca Ratón, USA (2001).

Delmoro, J., D. Muñoz, V. y otros tres autores, El Color en los Alimentos: Determinación de Color en Mieles, ISSN: 03293475, Invenio, 13(25), 145-152 (2010).

Ganganagowder, N. V. y P. Kamath, Intelligent Classification Models for Food Products Basis on Morphological, Color and Texture Features, doi: 10.15446/acag.v66n4.60049, Act. Agron., 66(4), 486-494 (2017).

Garzón-García, A. M., S. Dussán-Sarria y R. E. Melo-Sevilla, Estudio de la Variación de Parámetros de Color del Yacón Utilizando un Prototipo de Medición de Color y Temperatura, doi: 10.4067/S0718-07642018000600075, Inf. Tecnol., 29(6), 75-86 (2018).

Kirchner, E., P. Koeckhoven y S. Keshav, Improving Color Accuracy of Colorimetric Sensors, doi: 10.3390/s18041252, Sensors, 18(4), 1252 (2018).

Lohumi, S., H. Lee y otros seis autores, Calibration and Testing of a Raman Hyperspectral Imaging System to Reveal Powdered Food Adulteration, doi: 10.1371/journal.pone.0195253, PloS ONE,13(4), e0195253 (2018).

Mendoza, F., P. Dejmerk y J. M. Aguilera, Calibrated Color Measurements of Agricultural Foods Using Image Analysis, doi: 10.1016/j.postharvbio.2006.04.004, POSTHARVEST BIOL TEC., 41(3), 285-295 (2006).

Mery, D. y F. Pedreschi, Segmentation of Colour Food Images Using a Robust Algorithm, doi: 10.1016/j.jfoodeng.2004.04.001, J. Food Eng., 66(3), 353-360 (2005).

Newslow, D. Food Safety Management Programs: Applications, Best Practices, and Compliance, 188-191. CRC Press, Boca Ratón, USA, (2014).

Oblitas-Cruz, J. y W. Castro-Silupu, Computer Vision System for the Optimization of the Color Generated by the Coffee Roasting Process According to Time, Temperature and Mesh Size, doi: 10.11144/Javeriana.IYU18-2.cvso, Ingeniería y Universidad, 18(2), 355-368 (2014).

Padrón, P. C. A., P. M. G. León, H. A. I. Montes y G. R. A. Oropeza, Determinación del Color en Epicarpio de Tomates (Lycopersicum esculentum Mill.) con Sistema de Visión Computarizada Durante la Maduración, doi: 10.15517/RAC.V36l1.9969, Agron. Cost., 36(1), 97-111 (2012).

Rodríguez-Pulido, F. J., B. Gordillo, M. L. González-Miret y F. J. Heredia, Analysis of Food Appearance Properties by Computer Vision Applying Ellipsoids to Colour Data, doi: 10.1016/j.compag.2013.08.027, Comput. Electron. Agr., 99, 108115 (2013).

Romaniello, R., A. Leone y G. Peri, Measurement of Food Colour in $L^{*} a^{*} b^{*}$ Units from RGB Digital Image Using Least Squares Support Vector Machine Regression, doi: 10.4081/jae.2015.482, J. Ag. Eng., 46(4), 138-143 (2015).

Saldaña, E., R. Siche y otros cuatro autores, Computer Vision System in Real-Time for Color Determination on Flat Surface Food, doi: 10.17268/sci.agropecu.2013.01.06, Sci. agropecu., 4(1), 55-63 (2013).

Saldaña, E., R. Siche y otros tres autores, Measurement Parameter of Color on Yacon (Smallanthus sonchifolius) Slices Using a Computer Vision System, doi: 10.1016/j.Iwt.2014.06.037, LWT-Food Sci. Technol., 59(2), 1220-1226 (2014).

Tarlak, F., M. Ozdemir, y M. Melikoglu, Enfoque de Sistemas de Visión por Ordenador en las Mediciones de Color de los Alimentos: Parte I. Desarrollo de la Metodología, doi:10.1590/1678-457X.11615, Food Sci. Technol, Campinas, 36(2), 382-388 (2016).

Vallina, M. M., Tratamiento informático de la información, $1^{\text {a }}$ Ed., 333-334. Ediciones Paraninfo, Madrid, España, (2010). 